\title{
Down-regulation of radioresistance by LexA2 in Deinococcus radiodurans
}

\author{
Correspondence \\ Katsuya Satoh \\ sato.katsuya@jaea.go.jp
}

Received 16 May 2006

Revised 3 August 2006

Accepted 17 August 2006

\author{
Katsuya Satoh, ${ }^{1,2}$ Hirofumi Ohba, ${ }^{1,2}$ Haïtham Sghaier ${ }^{1,3}$ \\ and Issay Narumi ${ }^{1,2}$
}
1,2Gene Resource Research Group ${ }^{1}$ and DNA Repair Protein Group ${ }^{2}$, Quantum Beam Science Directorate, Japan Atomic Energy Agency, 1233 Watanuki, Takasaki 370-1292, Japan
${ }^{3}$ Material Science Laboratory, Department of Biological and Chemical Engineering, Faculty of Engineering, Gunma University, 1-5-1 Tenjin, Kiryu 376-8515, Japan

\section{INTRODUCTION}

Deinococcus radiodurans exhibits extraordinary resistance to the lethal and mutagenic effects of ionizing and UV radiation, as well as many other DNA-damaging agents. This resistance has been attributed to its highly proficient DNA repair capacity (Battista, 1997; Narumi, 2003; Cox \& Battista, 2005). The most noteworthy characteristic of $D$. radiodurans is its capacity for repairing DNA double-strand breaks induced by ionizing radiation (Dean et al., 1966; Kitayama \& Matsuyama, 1968; Grimsley et al., 1991). The highly efficient double-strand break repair process in $D$. radiodurans is radiation-inducible and is dependent on de novo protein synthesis following irradiation (Kitayama \& Matsuyama, 1971). Several DNA-damage-inducible proteins that may be necessary for DNA repair have been detected in cell extracts of $D$. radiodurans using 2D-PAGE analysis (Hansen, 1980; Tanaka et al., 1996). Recent transcriptome and proteome analyses have revealed the comprehensive expression profiles of radiation-inducible

Abbreviations: FL, firefly luciferase gene; RL, Renilla reniformis luciferase gene.

The GenBank/EMBL/DDBJ accession number for the lexA2 sequence reported in this paper is $A B 256032$. genes and proteins (Lipton et al., 2002; Liu et al., 2003; Tanaka et al., 2004; Zhang et al., 2005). It has therefore been established that $D$. radiodurans possesses a DNA damage response mechanism. Additionally, analysis of DNAdamage-sensitive strains IRS24 (Mattimore et al., 1995) and KH840 (Kitayama \& Matsuyama, 1975) identified a novel regulatory protein (DR0167; PprI protein, also called IrrE protein) that plays a role in regulating recA and $p p r A$ gene expression following $\gamma$-irradiation (Earl et al., 2002; Hua et al., 2003; Ohba et al., 2005). Furthermore, in a previous study we identified the radiation-responsive minimal promoter of the pprA gene encoding the novel radiation-inducible protein PprA, which plays a critical role in radioresistance of D. radiodurans (Narumi et al., 2004; Ohba et al., 2005).

RecA and LexA proteins in Escherichia coli play important roles in the DNA damage response repair mechanism (the SOS system) (Walker, 1984; Friedberg et al., 1995). The SOS response in Bacillus subtilis progresses in a similar manner, with $B$. subtilis RecA having an identical role in controlling the SOS regulon together with a cellular repressor protein that is functionally homologous to the E. coli LexA repressor (Wojciechowski et al., 1991). SOS-like processes have been conserved in a wide variety of eubacterial species (Miller \& 
Kokjohn, 1990; Roca \& Cox, 1990). On the one hand, RecA is the sole protein required for LexA1 (DRA0344) cleavage, although LexA1 is not involved in RecA induction in $D$. radiodurans (Narumi et al., 2001; Bonacossa de Almeida et al., 2002). The D. radiodurans DNA-repair-deficient mutant KI696 carrying the recA424 mutation was only slightly sensitive to $\gamma$-rays in comparison with the wild-type. RecA424 completely lacked the recombination activity but retained the co-protease activity. On the basis of this finding we suggested that the co-protease activity rather than the recombination activity of RecA contributed to the extraordinary radiation resistance of $D$. radiodurans (Satoh et al., 2002). Bioinformatic analysis of the D. radiodurans genome sequence showed that the genome encodes a second, diverged copy of LexA1, LexA2 (DRA0074), which retains the same arrangement of the helix-turn-helix DNA-binding domain and the autocleavage domain (Makarova et al., 2001). Sheng et al. (2004) suggested that the product of gene dra0074 is involved in metabolic pathways other than the DNA repair response elicited by irradiation. However, the exact role of LexA2 and the relationship between LexA1 and LexA2 in the radiation response are poorly understood. The biochemical function of LexA2 therefore requires further clarification.

This study began with the purpose of elucidating the role of LexA2 in relation to the radiation response mechanism of $D$. radiodurans. To accomplish this purpose, we generated lexA1 and lexA2 disruptant strains in wild-type and recA backgrounds. Our results suggest that LexA2 contributes to the regulation of the radiation response promoter of the pprA gene in D. radiodurans and that it is not involved in RecA induction.

\section{METHODS}

Bacterial strains, cosmids, plasmids and growth conditions. The bacterial strains and plasmids used in this study are listed in Table 1. D. radiodurans cells were grown with agitation at $30{ }^{\circ} \mathrm{C}$ in TGY broth, containing $0.5 \%(\mathrm{w} / \mathrm{v})$ tryptone-peptone, $0.1 \%(\mathrm{w} / \mathrm{v})$ glucose and $0.3 \%(\mathrm{w} / \mathrm{v})$ yeast extract, or on TGY agar solidified with $1.5 \%(\mathrm{w} / \mathrm{v})$ agar. When necessary, $3 \mu \mathrm{g}$ chloramphenicol $\mathrm{ml}^{-1}$, $16 \mu \mathrm{g}$ kanamycin $\mathrm{ml}^{-1}$ and $350 \mu \mathrm{g}$ spectinomycin $\mathrm{ml}^{-1}$ were added. E. coli strains were grown in Luria-Bertani (LB) broth-Miller or on LB agar-Lennox at $37^{\circ} \mathrm{C}$. Selective drug concentrations for E. coli were $50 \mu \mathrm{g}$ ampicillin $\mathrm{ml}^{-1}, 34 \mu \mathrm{g}$ chloramphenicol ml $\mathrm{m}^{-1}$ and $25 \mu \mathrm{g}$ kanamycin $\mathrm{ml}^{-1}$.

Construction of gene disruptant strains. In an effort to gain an insight into the role of LexA2 in the radiation response mechanism, we generated $\operatorname{rec} A$, lexA1 and lexA2 disruptant strains within a wildtype background. The disruptants were generated using a previously described directed insertional mutagenesis technique (Funayama et al., 1999; Narumi et al., 2001; Nishida \& Narumi, 2002). The previously constructed disruption plasmid pKSCR3 (carrying the recA229: : cat mutation) (Narumi et al., 2001) was used to disrupt the recA gene. To construct plasmids carrying the lexA1 null mutation, a 975 bp HincII fragment (KatAPH cassette) from pKatAPH3 (Ohba et al., 2005) or a 948 bp HincII fragment (KatAAD cassette) from pKatAAD2, which is a spectinomycin-resistant version of pKatAPH3, was ligated to EagI-digested pZA8 (Kikuchi et al., 1999).
The resulting plasmids were designated pLKN2 (carrying the lexA1166::aph mutation) and pLAN2 (carrying the lexA1-166::aad mutation), respectively.

pDC201 is a cosmid clone of the genome library (Narumi et al., 1997), and it was used to clone the D. radiodurans lexA2 gene. The $5.6 \mathrm{~kb}$ EcoRI fragment carrying the D. radiodurans lexA2 locus was isolated from pDC201 and subcloned in the EcoRI site of pUC19. The resulting plasmid was designated pLE44. Plasmid pLE44 was digested with BsrGI and HindIII, blunted with T4 DNA polymerase, and then self-ligated to yield plasmid pLEB7. Plasmid pLEB7 was subjected to site-directed mutagenesis to create a unique SmaI site in lexA2 ( $\mathrm{T}$ at nucleotide position 365 was changed to G). Site-directed mutagenesis was carried out using the QuikChange Site-directed Mutagenesis kit (Stratagene) with primers T365G-F (5'-GTGCAGCAGGTCCCGGGCTACATCGCCGGTCAG-3') and T365G-R (5'-CTGACCGGCGATGTAGCCCGGGACCTGCTGCAC- $3^{\prime}$ ), and the resulting plasmid was designated pLSM2. The KatAPH cassette was ligated to SmaI-digested pLSM2 to construct plasmids carrying the lexA2 null mutation, and the resulting plasmid was designated pLK2N2 (carrying the lexA2-362::aph mutation).

All disruptant strains were generated from $D$. radiodurans $\mathrm{R}_{1}$ (ATCC 13939). The recA229::cat, lexA1-166::aph and lexA2-362::aph mutations were introduced into the genome of $D$. radiodurans and the resulting disruptant strains were designated XRA1 (recA disruptant), XLK1 (lexA1 disruptant) and XL2K1 (lexA2 disruptant), respectively. We introduced the lexA1 null mutation into the $r e c A$ disruptant strain and the resulting $r e c A$ lexA1 double-disruptant strain was designated XRL11. Additionally, the recA null mutation was introduced into the lexA2 disruptant strain to yield the recA lexA2 double-disruptant strain XL2R8, and the lexA1 null mutation was introduced into the lexA2 disruptant strain to yield the lexA2 lexA1 double-disruptant strain WXL1.

Construction of expression plasmid and DNA sequencing of the $D$. radiodurans lexA2 gene. To isolate the lexA2 coding region, PCR was carried out using PfuTurbo HotStart DNA polymerase (Stratagene) with pLEB7 and specific oligonucleotides A74EX-F3 (5'-CTCAGTCTAATTCGCATATGCGAACATTTC- $3^{\prime}$ ) and A74EXR2 (5'-GCAGGGATCCGGGCAGAGGCTCAGGC-3'), possessing $N d e \mathrm{I}$ and Bam $\mathrm{HI}$ restriction sites (underlined in the sequences), respectively. PCR products were then digested with NdeI and BamHI to adapt the termini for in-frame insertion of lexA2 into the NdeI$B a m H I$ sites in the pET3a vector (Novagen). The resulting expression plasmid was designated pET3lexA2. The DNA sequence of the expression plasmids was checked to confirm the absence of errors by PCR using T7 promoter primer ( $5^{\prime}$-TAATACGACTCACTATAGGG- $\left.3^{\prime}\right)$ and T7 terminator primer ( $5^{\prime}$-GCTAGTTATTGCTCAGCGG-3').

To check the sequence of the lexA2 locus in plasmid pLEB7 and the D. radiodurans genome, direct sequencing was carried out using the oligonucleotide primer sets A74DI-F (5' -ATGGCCTGATCGCAGAACGTATG-3') and A74DI-R (5'-TGTCACTCCCTGAAAGGTGCTAGA-3').

RNA isolation and primer extension assay. D. radiodurans cells were grown in TGY broth at $30^{\circ} \mathrm{C}$ with agitation to early stationary phase. Total RNA was isolated from the harvested cells using a FastRNA Pro Blue Kit (Qbiogene) with a DNA-free kit (Ambion). The specific oligonucleotide IRD800lexA2 (5'-CCTTCGCCGCGCTGGTAGCAAG- $3^{\prime}$ ) was used to map the transcriptional start site of the lexA2 mRNA. RNA $(10 \mu \mathrm{g})$ and the IRD800 infrared dye-labelled primer $(1 \mathrm{pmol})$ were incubated in a buffer containing $10 \mathrm{mM}$ Tris/

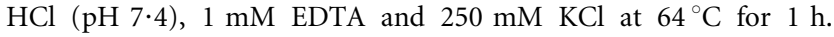
After annealing, reverse transcription was carried out using Superscript III RNase $\mathrm{H}^{-}$reverse transcriptase (Invitrogen) as recommended by the manufacturer. Analysis of the primer extension product was performed using a DNA Analyser model 4200-1G 
Table 1. Strains, cosmids and plasmids

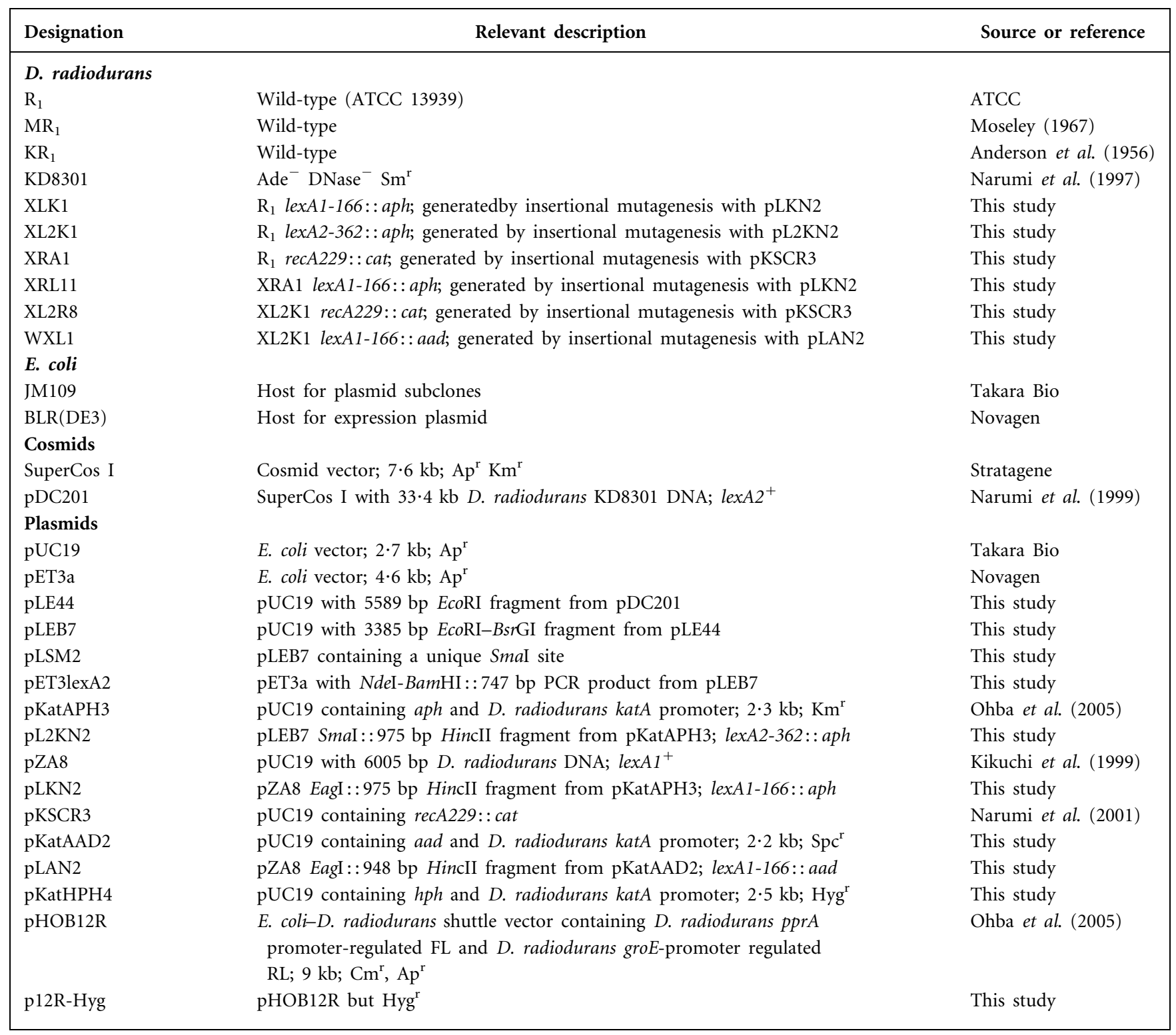

(LI-COR). The size of the primer extension product was determined by comparison with the DNA sequence generated using the same primer with plasmid pLEB7 as a template.

Protein purification. E. coli $\mathrm{BLR}(\mathrm{DE} 3)$ carrying pET3lexA2 was cultivated in LB broth-Miller containing ampicillin. At an $\mathrm{OD}_{600}$ of approximately $0 \cdot 5$, IPTG was added to a final concentration of $0.4 \mathrm{mM}$ and growth was continued for an additional $2 \mathrm{~h}$. The cells were then harvested by centrifugation. The cell pellet was resuspended in cold buffer P1 (20 mM sodium phosphate $\mathrm{pH} 6.0$ and $0 \cdot 1 \mathrm{mM}$ EDTA) containing $1 \mathrm{mM}$ PMSF and $0 \cdot 1 \%(\mathrm{w} / \mathrm{v})$ protease inhibitor cocktail (Calbiochem) in $1 / 100$ of the original culture volume, and stored at $-80^{\circ} \mathrm{C}$. All subsequent steps were carried out at $4{ }^{\circ} \mathrm{C}$ unless otherwise indicated.

Cells were allowed to thaw with occasional stirring and the suspension was sonicated for $10 \mathrm{~min}$ on ice; debris was removed by centrifugation at $10000 \mathrm{~g}, 4{ }^{\circ} \mathrm{C}$ for $30 \mathrm{~min}$. The supernatant was then applied to a HiPrep 16/10 Heparin FF column (GE Healthcare) equilibrated with buffer P1. The column was washed with four column volumes of buffer $\mathrm{P} 1$. Following elution with 10 volumes of a linear $\mathrm{NaCl}$ gradient $(0$ to $1 \mathrm{M}$ ), the fractions containing LexA2 were pooled. The majority of LexA2 was eluted at approximately $0.6 \mathrm{M} \mathrm{NaCl}$. The pooled fractions were concentrated and desalted using an Amicon Ultra-15 filter unit (Millipore) with buffer P1, and the fraction was then loaded onto a Mono S HR 5/5 column (GE Healthcare) equilibrated with buffer P1. The column was washed with 10 column volumes of buffer P1. Following elution with 20 volumes of a linear $\mathrm{NaCl}$ gradient $(0 \cdot 3$ to $1 \mathrm{M})$, the fractions containing LexA2 were pooled. The majority of LexA2 was eluted at approximately $0.5 \mathrm{M} \mathrm{NaCl}$. The pooled fractions were concentrated using an Amicon Ultra-15 filter unit with buffer P1.

Autodigestion and RecA-mediated cleavage reaction. The autodigestion reaction was assayed by incubating $8 \mu \mathrm{M}$ LexA2 in $50 \mathrm{mM}$ Tris/ $\mathrm{HCl}(\mathrm{pH} 10 \cdot 0)$ at $37^{\circ} \mathrm{C}$ for $16 \mathrm{~h}$. For the cleavage of LexA2 mediated by $D$. radiodurans $\mathrm{RecA}$, the reaction mixture contained $20 \mathrm{mM}$ Tris/HCl ( $\mathrm{pH} 6 \cdot 0$ ), $10 \mathrm{mM} \mathrm{MgCl}_{2}, 6 \mu \mathrm{M}$ oligonucleotide (5'-GAGAACCATGGATGGACCAATAATAATGACTAGAG-3'; 
35-mer), $1 \mathrm{mM} \mathrm{ATP} \gamma \mathrm{S}, 4 \cdot 2 \mu \mathrm{M}$ RecA (Satoh et al., 2002) and $8 \mu \mathrm{M}$ LexA2. To activate RecA by binding with ATP $\gamma$ S and oligonucleotide, the mixture without LexA2 was first combined in a microtube and preincubated at $37^{\circ} \mathrm{C}$ for $10 \mathrm{~min}$. The reaction was then initiated by addition of LexA2, and the reaction mixture was incubated at $37^{\circ} \mathrm{C}$ for $1 \mathrm{~h}$. After quenching by addition to SDS sample buffer and heating at $95^{\circ} \mathrm{C}$ for $10 \mathrm{~min}$, the reaction products were subjected to $15 \%$ (w/v) SDS-PAGE. LexA2 and breakdown products were visualized by staining with GelCode Blue Stain Reagent (Pierce).

Detection of intracellular levels of $D$. radiodurans RecA, LexA1 and LexA2. D. radiodurans cells were grown in TGY broth at $30^{\circ} \mathrm{C}$ with agitation to early stationary phase. Cells were harvested by centrifugation at $5000 \mathrm{~g}, 4{ }^{\circ} \mathrm{C}$ for $5 \mathrm{~min}$, washed twice with $10 \mathrm{mM}$ sodium phosphate buffer $(\mathrm{pH} 7 \cdot 0)$ and resuspended in the same buffer. Aliquots $(20 \mathrm{ml})$ of the cell suspension were irradiated at room temperature for $1 \mathrm{~h}$ with ${ }^{60} \mathrm{Co} \gamma$-rays at a dose rate of $2 \mathrm{kGy} \mathrm{h}^{-1}$. The cells were then centrifuged at $5000 \mathrm{~g}, 4{ }^{\circ} \mathrm{C}$ for $5 \mathrm{~min}$ and resuspended in $200 \mathrm{ml}$ TGY broth. The cell suspension was incubated at $30^{\circ} \mathrm{C}$ for different post-irradiation times $(0,0 \cdot 5,1$ and $2 \mathrm{~h})$. Aliquots $(80 \mathrm{ml})$ of the culture were then harvested as needed. To obtain crude protein extract, the cells were resuspended in lysis buffer $(0.5 \mathrm{ml})$ containing $50 \mathrm{mM}$ Tris/ $\mathrm{HCl}(\mathrm{pH} \mathrm{7} \cdot 4), 5 \mathrm{mM}$ EDTA, $1 \mathrm{mM}$ PMSF, $0 \cdot 1 \%(\mathrm{w} / \mathrm{v})$ protease inhibitor cocktail (Calbiochem) and $1 \%(\mathrm{w} / \mathrm{v})$ SDS, and disrupted using a FastPrep Cell Disruptor FP120 (Savant Instruments) with a FastProtein Blue Kit (Qbiogene). Following removal of cell debris by centrifugation, the supernatant was subjected to 12.5 or $15 \%(\mathrm{w} / \mathrm{v})$ SDS-PAGE. The resolved proteins were transferred onto a PVDF membrane (Millipore). D. radiodurans RecA, LexA1 or LexA2 were detected using $D$. radiodurans RecA antibody (diluted $1: 10000), D$. radiodurans LexA1 antibody (diluted 1:10000) (Narumi et al., 2001) or D. radiodurans LexA2 antibody (diluted 1:4000) together with alkaline-phosphatase-conjugated anti-mouse or anti-rabbit IgG antisera (Applied Biosystems). The $D$. radiodurans RecA and LexA2 antibodies (mouse, polyclonal) were raised against purified D. radiodurans RecA (Satoh et al., 2002) and purified $D$. radiodurans LexA2, respectively, at ImmunoBiological Laboratories (Gunma, Japan). Chemiluminescent signals on the PVDF membrane were visualized using a Lumi-Imager F1 Workstation (Roche Diagnostics). D. radiodurans LexA1 and LexA2 antibodies did not cross-react with each antigen.

Measurement of cell survival rate. D. radiodurans cells were grown, harvested, washed and resuspended as described above. Aliquots $(0 \cdot 1 \mathrm{ml})$ of the cell suspension were dispensed into test tubes and irradiated at room temperature for 1 to $3 \mathrm{~h}$ with ${ }^{60} \mathrm{Co} \gamma$ rays at dose rates from $0 \cdot 1$ to $5 \mathrm{kGy} \mathrm{h}^{-1}$ that were regulated by changing the distance of the samples from the $\gamma$-ray source. Irradiated samples were appropriately diluted with $10 \mathrm{mM}$ phosphate buffer, spread onto TGY agar, and incubated at $30^{\circ} \mathrm{C}$ for 3 days prior to the enumeration of colonies.

Luciferase reporter assay. The luciferase reporter plasmid pHOB12R contained engineered firefly luciferase gene (FL), Renilla reniformis luciferase gene (RL) and the chloramphenicol resistance gene as a genetic marker. The expression of FL and RL was regulated by the $D$. radiodurans pprA and groE minimal promoters, respectively (Ohba et al., 2005). The luciferase reporter plasmid p12R-Hyg, which carries a hygromycin-resistance gene instead of a chloramphenicol-resistance gene, was constructed as a pHOB12R-based plasmid. A 1199 bp HincII fragment (KatHPH cassette) from pKatHPH4, which is a hygromycin-resistant version of pKatAPH3, was ligated to MscI- and FspI-digested pHOB12R. D. radiodurans cells carrying the resulting plasmid p12R-Hyg were grown, harvested, washed and resuspended as described above. Aliquots $(1 \mathrm{ml})$ of the cell suspension were irradiated at room temperature for $2 \mathrm{~h}$ with ${ }^{60} \mathrm{Co} \gamma$-rays at a dose rate of $4 \mathrm{kGy} \mathrm{h}^{-1}$. The cells were then centrifuged at $5000 \mathrm{~g}, 4^{\circ} \mathrm{C}$ for $5 \mathrm{~min}$, and resuspended in $4 \mathrm{ml}$ TGY broth. The cell suspension was incubated at $30{ }^{\circ} \mathrm{C}$ for different post-irradiation incubation times, with agitation. FL and RL activities in cells $(20 \mu \mathrm{l})$ were measured using the Dual-Glo Luciferase Assay System (Promega). Chemiluminescent signals were visualized using a LumiImager F1 Workstation. The relative reporter activity was calculated as the ratio of the luminescence from FL (experimental reporter) to the luminescence from RL (control reporter).

\section{RESULTS}

\section{Sequence and structure of $D$. radiodurans IexA2}

To construct the disruptant plasmid carrying the lexA2 null mutation, we tried digesting plasmid pLEB7 with Aor51HI, based on the DRA0074 sequence deposited in the public database (GenBank accession no. AE001862). However, pLEB7 surprisingly could not be digested with Aor $51 \mathrm{HI}$. We therefore decided to check the nucleotide sequence of the lexA2 locus in pLEB7. We found that there is an additional cytosine nucleotide (nucleotide position 612 in Fig. 1) that was not present in the DRA0074 sequence. Furthermore, the lexA2 loci in $D$. radiodurans strains $\mathrm{MR}_{1}, \mathrm{KR}_{1}$ and $\mathrm{R}_{1}$, which are different isolates of the wild-type strain (Islam et al., 2003), were resequenced using primers A74DI-F and A74DI-R. In each case, the sequence was identical to our lexA2 sequence, suggesting an error in the previous sequence. We deposited our lexA2 sequence in GenBank under accession no. AB256032. The addition of one nucleotide affected the open reading frame; 44 amino acid residues were altered, and the predicted amino acid sequence of lexA2 was 8 amino acid residues longer than the previous sequence (Fig. 1). Fig. 2 shows the alignment of the amino acid sequence of E. coli LexA and D. radiodurans LexA1 and LexA2. Although $D$. radiodurans LexA2 exhibited low similarity to LexA of $E$. coli and $D$. radiodurans $(23.4 \%$ and $24 \cdot 4 \%$ identity, respectively), LexA2 retained the amino acid residues involved in selfcleavage of the protein (Ala-130, Gly-131, Ser-163 and Lys203) and a helix-turn-helix DNA-binding motif (Ile-38 to Leu-100) (Fig. 2). The C-terminal region of E. coli LexA (Phe-186 to Arg-197) participates in dimeric interactions (Fig. 2) (Luo et al., 2001). The C-terminal region of LexA2 revealed in this study may also be involved in dimeric interactions in a manner similar to E. coli LexA. The amino acid sequence alignment shows that $D$. radiodurans LexA2 has a longer N-terminal sequence than $E$. coli and $D$. radiodurans LexA1 proteins (Fig. 2). The primer extension assay revealed that the transcription start site of lexA2 was located at -97 and -98 upstream of the translation start site (Fig. 3). No promoter-like sequence was found between the translation start and transcription start sites for lexA2. These results support the existence of an extended $\mathrm{N}$-terminal region in $D$. radiodurans LexA2.

\section{Autodigestion and RecA-mediated cleavage}

The purified $D$. radiodurans LexA2 migrated on SDSpolyacrylamide gel with an apparent molecular mass of 


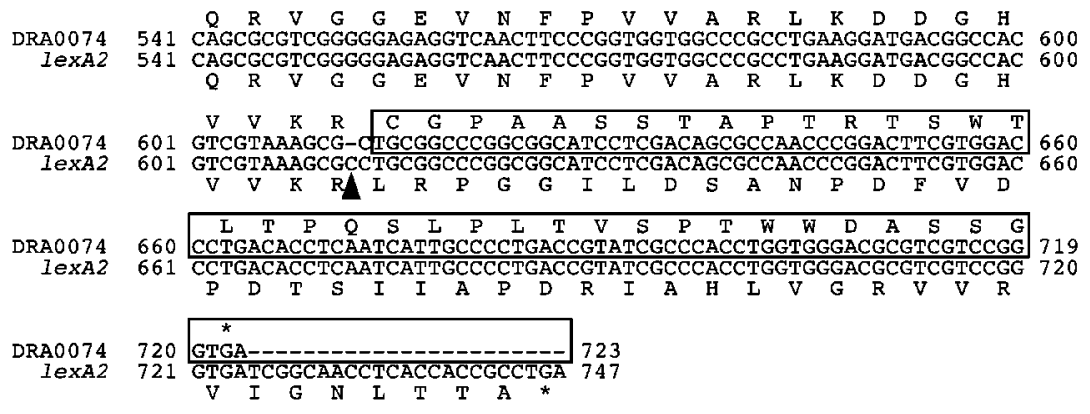

Fig. 1. Nucleotide sequence of the $D$. radiodurans lexA2 gene. The predicted amino acid sequence is shown above the nucleotide sequence of DRA0074 described in a previous study (White et al., 1999) in single-letter code. The predicted amino acid sequence of $D$. radiodurans lexA2 determined in this study is shown below the nucleotide sequence. Numbers on both sides represent the coordinates of each sequence. The filled triangle indicates the nucleotide that differs between the two sequences. The reading frame affected by the previous error is shown in boxes.

approximately $26 \mathrm{kDa}$ (Fig. 4a). This was very close to the molecular mass (26750 Da) calculated from the amino acid sequence of LexA2.

As described above, LexA2 retained the amino acid residues involved in self-cleavage by the amino acid alignment (Fig. 2). Similar to E. coli LexA, D. radiodurans LexA1 undergoes autodigestion when subjected to alkaline conditions and RecA-mediated cleavage (Narumi et al., 2001). We investigated whether these proteolytic cleavage properties are conserved in D. radiodurans LexA2. Purified LexA2 was autodigested at $\mathrm{pH} 10$ to yield two breakdown products (Fig. 4b, lane 2). LexA2 was cleaved by incubation with $D$. radiodurans RecA to produce two breakdown products whose sizes are the same as those observed in autodigestion (Fig. 4b, lane 6). No breakdown product was observed when RecA, oligonucleotide or ATP $\gamma \mathrm{S}$ was omitted from the reaction (Fig. $4 \mathrm{~b}$, lanes 3 to 5). These results support the conclusion that $D$. radiodurans LexA2 maintains proteolytic activity that can be stimulated by $D$. radiodurans RecA.

\section{Changes in intracellular RecA, LexA1 and LexA2 levels in disruptants following irradiation}

To determine if LexA2 is involved in RecA induction and whether cleavage of LexA2 is promoted by RecA in vivo, changes in RecA, LexA1 and LexA2 levels following $\gamma$ irradiation $(2 \mathrm{kGy})$ were compared between parental and disruptant strains.

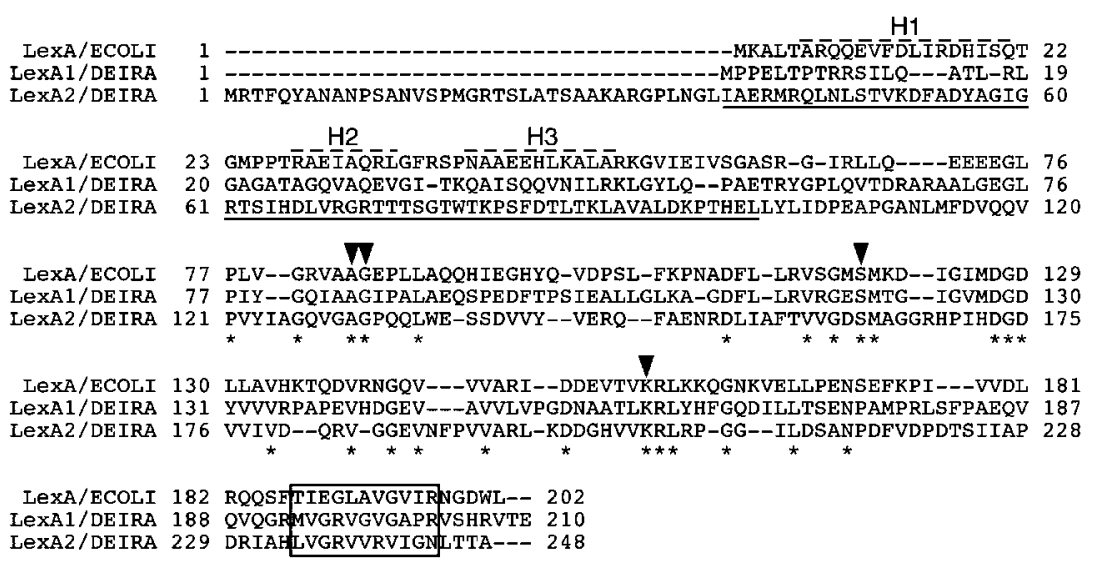

Fig. 2. Multiple amino acid sequence alignment of $E$. coli LexA (Horii et al., 1981) and D. radiodurans LexA1 (Narumi et al., 2001) and LexA2 (this study). Multiple alignment was determined using CLUSTAL W (Thompson et al., 1994). Dashes indicate gaps in the alignment. Numbers on both sides represent the coordinates of each protein. Asterisks indicate identical residues. Filled triangles indicate conserved Ala and Gly residues in the cleavage site, as well as Ser and Lys residues required for cleavage. The locations of three $\alpha$-helices $(\mathrm{H} 1, \mathrm{H} 2$ and $\mathrm{H} 3)$ found in $E$. coli LexA are shown according to the description of Fogh et al. (1994). The underline indicates the helix-turn-helix DNA-binding motif of $D$. radiodurans LexA2 that was detected using the Search Motif program (Bateman et al., 2004). The box indicates the region of dimeric interactions in $E$. coli LexA (Luo et al., 2001). 


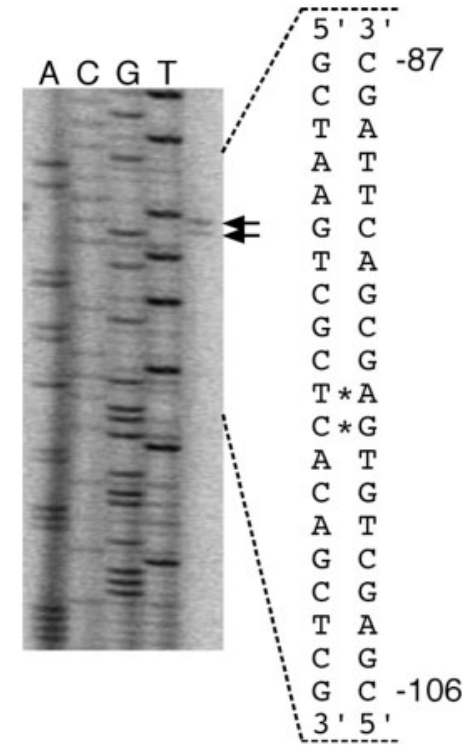

Fig. 3. Transcription start site of lexA2 determined by primer extension analysis. Sequence ladders of the $5^{\prime}$-flanking region of lexA2 primed with the same primer as used in the primer extension reaction are shown in lanes $A, C, G$ and $T$. The arrows indicate the primer extension products. Transcription start sites are marked with asterisks.

Fig. 5 shows the results of experiments in which cells treated with or without radiation were incubated in fresh TGY broth for different post-irradiation incubation times and then subjected to Western blot analysis using D. radiodurans RecA, LexA1 or LexA2 antibodies. The intracellular level of RecA increased in the lexA1 disruptant strain following $\gamma$ irradiation as in the wild-type strain (Fig. 5a). The LexA1 level in the recA disruptant strain did not change for any of the post-irradiation incubation times examined (Fig. 5b). These results confirmed our previous conclusions that $D$. radiodurans LexA1 is not involved in RecA induction following $\gamma$-irradiation and that RecA is the sole protein required for LexA1 cleavage (Narumi et al., 2001). RecA induction following $\gamma$-irradiation was also observed in the lexA2 disruptant and lexA1 lexA2 double-disruptant strains (Fig. 5a), clearly indicating that neither LexA1 nor LexA2 is involved in RecA induction. While a decreased level of LexA2 was observed in wild-type and lexA1 disruptant strains following $\gamma$-irradiation for all post-irradiation incubation times (Fig. 5c), $\gamma$-irradiation did not affect the level of LexA2 in the recA disruptant strain. This result indicated that RecA is the sole protein required for LexA2 cleavage, and supported our observation of in vitro RecAmediated LexA2 cleavage (Fig. 4b).

In the wild-type strain, the LexA1 level decreased to half the initial level at $1 \mathrm{~h}$ following irradiation, and increased to $62 \%$ of the initial level $2 \mathrm{~h}$ following irradiation (Fig. 5b). Surprisingly, the LexA1 level in the lexA2 disruptant strain almost recovered $1 \mathrm{~h}$ after irradiation, although the decrease

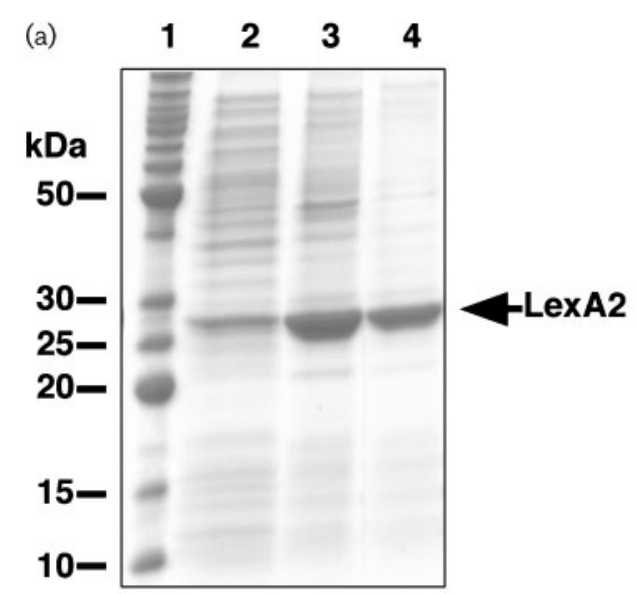

(b)

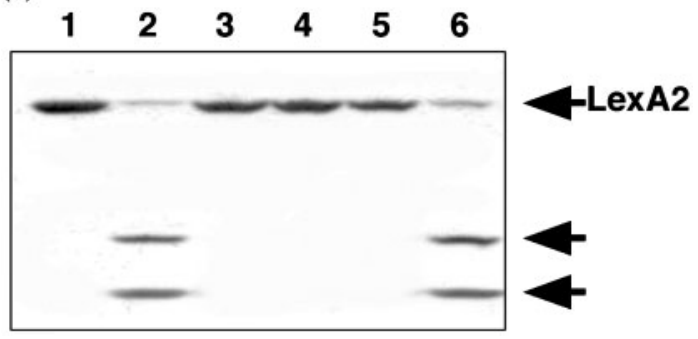

Fig. 4. D. radiodurans LexA2 protein. (a) Purification of LexA2. Samples were subjected to $15 \%$ SDS-PAGE and stained with GelCode Blue Stain Reagent. Lane 1, $10 \mathrm{kDa}$ protein ladder (Invitrogen); lane 2, total cellular proteins from $E$. coli $\mathrm{BLR}(\mathrm{DE} 3) / \mathrm{pET} 3$ lexA2wt induced by IPTG; lane 3, pooled LexA fractions from HiTrap Heparin HP column; lane 4, pooled LexA fractions from Mono $S$ column. The arrow on the right indicates the position of the $26 \mathrm{kDa}$ band of LexA2. (b) Autodigestion and RecA-mediated cleavage of purified LexA2. LexA2 was incubated in $50 \mathrm{mM}$ Tris/ $\mathrm{HCl}(\mathrm{pH} \mathrm{10.0)}$ for $\mathrm{Oh}$ (lane 1) and $16 \mathrm{~h}$ (lane 2). For RecA-mediated cleavage, LexA2 was incubated in the absence or presence of $D$. radiodurans $R e c A$, oligonucleotide (5'-GAGAACCATGGATGGACCAATAATAATGACTAGAG-3'; 35-mer) and ATP $\gamma \mathrm{S}$ in a buffer containing $20 \mathrm{mM}$ Tris/ $\mathrm{HCl}(\mathrm{pH} 6 \cdot 0), 10 \mathrm{mM} \mathrm{MgCl}{ }_{2}$ at $37^{\circ} \mathrm{C}$ for $1 \mathrm{~h}$, and subjected to $15 \%(w / v)$ SDS-PAGE. LexA2 and breakdown products were visualized by staining with GelCode Blue Stain Reagent. Lane 3, RecA was omitted; lane 4, oligonucleotide was omitted; lane $5, \operatorname{ATP} \gamma \mathrm{S}$ was omitted; lane 6 , complete reaction. Arrows indicate the positions of breakdown products of LexA2.

in the LexA1 level was observed until $0.5 \mathrm{~h}$ in the case of the wild-type strain (Fig. 5b). The damaged DNA leads to the production of segments of ssDNA that act as a substrate for RecA activation. As in E. coli, the co-protease of $D$. radiodurans RecA could not activate in the absence of ssDNA (Fig. 4b, lane 4). The segments of ssDNA disappear as a consequence of various DNA repair processes. Since the decrease in the intracellular LexA1 level reflects the coprotease activity of RecA, the recovery to a basal level of LexA1 indicates the inactivation of RecA (Fig. 5b). This 


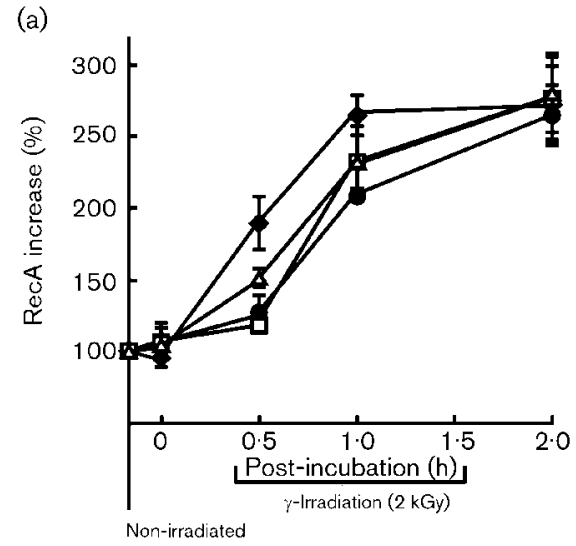

(b)

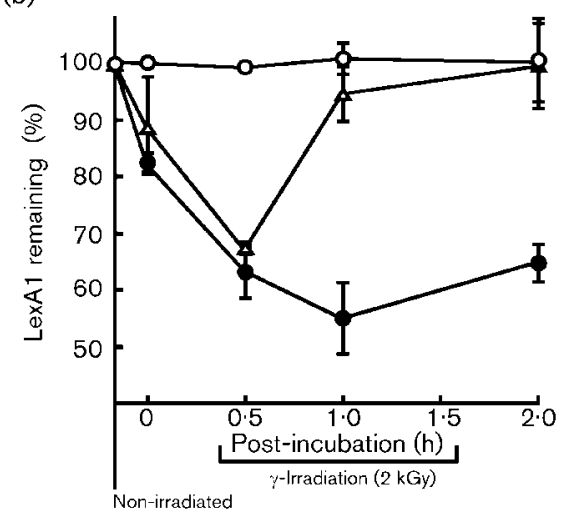

(c)

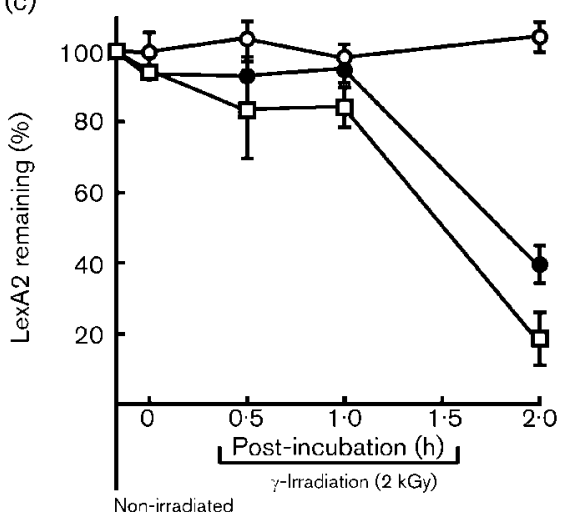

Fig. 5. Changes in intracellular RecA, LexA1 and LexA2 levels following irradiation. Cells were irradiated with $\gamma$-rays at a dose of $2 \mathrm{kGy}$. Dots indicate the integrated density of chemiluminescent signals by Western blotting at the indicated times. Each point represents the mean $\pm S D$ of three independent experiments. (a) RecA increase. (b) LexA1 remaining. (c) LexA2 remaining. - Wild-type; $\bigcirc, \operatorname{rec} A$ disruptant strain; $\square$, lexA1 disruptant strain; $\triangle$, lexA2 disruptant strain; $\diamond$ lexA1 lexA2 double-disruptant strain.

result suggested that the inactivation of RecA was faster in the lexA2 disruptant strain than in the wild-type strain. Given that the inactivation of RecA is associated with the completion of DNA repair, the DNA repair capacity might be enhanced in the lexA2 disruptant strain relative to the wild-type strain.

\section{Sensitivity of gene disruptant strains to $\gamma$-rays}

To investigate DNA repair capacities, we examined the sensitivity of the disruptant strains to $\gamma$-rays. As shown in Fig. 6(a), the lexA2 disruptant and lexA1 lexA2 doubledisruptant strains exhibited a much higher resistance than the wild-type strain at high doses ( 8 to $15 \mathrm{kGy}$ ) of $\gamma$-rays. The $\mathrm{LD}_{90}$ values $(12 \cdot 7 \mathrm{kGy})$ of the lexA2 disruptant and lexA1 lexA2 double-disruptant strains were $1 \cdot 6$-fold higher than those for the wild-type strain $(7 \cdot 8 \mathrm{kGy})$. On the other hand, the radioresistance of the lexA 1 disruptant strain was equal to that of the wild-type strain.

We also examined the effect of lexA1 and lexA2 disruption on radioresistance in a $\operatorname{rec} A$ background. As shown in Fig. 6(b), the recA disruptant strain exhibited extreme sensitivity ( $\mathrm{LD}_{90}$ value $0.09 \mathrm{kGy}$ ). In a $\operatorname{rec} A$ background, neither lexA1 nor lexA2 disruption altered the radiosensitivity of $D$. radiodurans ( $\mathrm{LD}_{90}$ values 0.09 and $0 \cdot 12 \mathrm{kGy}$, respectively).

\section{Change in pprA promoter activation in disruptants following $\gamma$-irradiation}

The pprA gene encoding the novel radiation-inducible protein PprA plays a critical role in the radioresistance of $D$. radiodurans (Narumi et al., 2004). To investigate whether LexA homologues are involved in PprA induction, changes in $\operatorname{ppr} A$ promoter activation following $\gamma$-irradiation ( $8 \mathrm{kGy}$ ) were compared between parental and disruptant strains using the luciferase reporter assay. pprA promoter activation reached a maximum at $3 \mathrm{~h}$ post-irradiation incubation in all of the strains (Fig. 7). Remarkably, the maximum level of pprA promoter activation in the lexA2 disruptant strain was approximately $1 \cdot 4$-fold higher than that recorded for the wild-type strain. The change in $p p r A$ promoter activation in the lexA1 disruptant strain was very similar to that of the wild-type strain. On the other hand, the maximum level of pprA promoter activation in recA disruptant strains was lower than that of the wild-type strain. The maximum level of pprA promoter activation of the recA lexA2 doubledisruptant strain was slightly higher than that recorded for the recA disruptant strain. These results indicated that the $p p r A$ promoter activation was enhanced by lexA2 disruption.

\section{DISCUSSION}

In this study, we resequenced the lexA2 sequence of $D$. radiodurans $\mathrm{R}_{1}$ (Fig. 1) and found a single base difference between our sequence and the published $D$. radiodurans $\mathrm{R}_{1}$ genome sequence (White et al., 1999). It was reported recently that the $D$. radiodurans strain used in the genome project was not the $\mathrm{R}_{1}$ type strain that had been deposited in the American Type Culture Collection as ATCC no. 13939, but that it was the strain designated BAA- 816 in which it was 

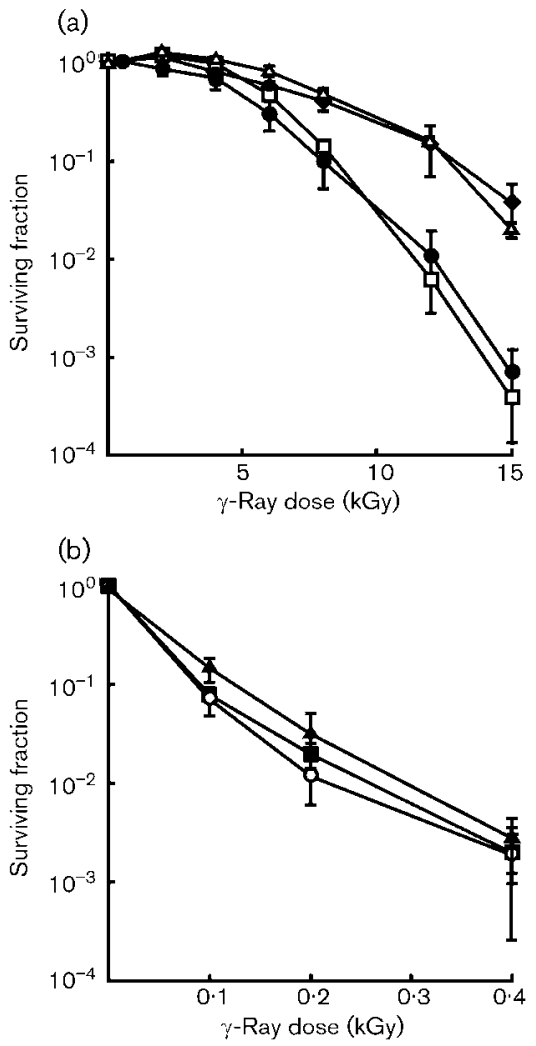

Fig. 6. Sensitivity of $\operatorname{rec} A$, lexA1 and lexA2 disruptant strains to $\gamma$-rays. Each point represents the mean \pm SD of three independent experiments. (a) $\bullet$, Wild-type; $\square$, lexA1 disruptant strain; $\triangle$, lexA2 disruptant strain; $>$, lexA1 lexA2 double-disruptant strain. (b) $\bigcirc$, recA disruptant strain; $\mathbf{\square}, \operatorname{rec} A$ lexA1 double-disruptant strain; $\boldsymbol{\Lambda}$, recA lexA2 double-disruptant strain.

suspected many mutations were acquired during years of separate culture (Mennecier et al., 2004). Although resequencing of the lexA2 locus in this study revealed the correct sequence, the fact remains that $D$. radiodurans contained a second, diverged copy of lexAl.

We purified $D$. radiodurans LexA2 to obtain a molecular mass of approximately $26 \mathrm{kDa}$ (Fig. 4a), which was smaller than the molecular mass (approx. $33 \mathrm{kDa}$ ) of $D$. radiodurans DRA0074 purified by Sheng et al. (2004). For this reason it was thought that the lexA2 stop codon of the expression plasmid pET3lexA2wt constructed in this study differed from the expression plasmid constructed by Sheng et al. (2004) because they had designed PCR primers for amplifying the dra0074 gene on the basis of the published sequence (White et al., 1999). Consequently, the DRA0074 protein purified by Sheng et al. (2004) may contain amino acid residues that originated from a vector at its C-terminal region.

The N-terminal region of E. coli LexA is involved in DNAbinding activity (Fogh et al., 1994). The purification of $D$. radiodurans LexA2 showed a heparin column to be very effective (Fig. 4a), suggesting that LexA2 retains

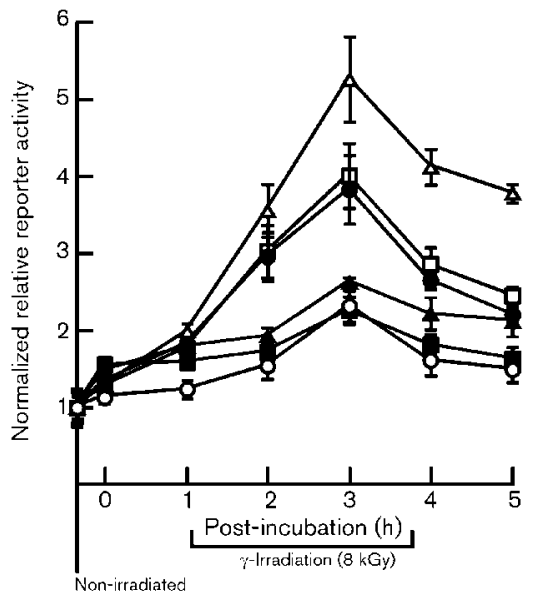

Fig. 7. Changes in pprA promoter activation following irradiation. Cells were irradiated with $\gamma$-rays at a dose of $8 \mathrm{kGy}$. The relative reporter activity was calculated as the ratio of the luminescence from the experimental reporter (FL) to the luminescence from the control reporter $(\mathrm{RL})$ at the indicated times. Each point represents the mean $\pm S D$ of three independent experiments. Wild-type; $\square$, lexA1 disruptant strain; $\triangle$, lexA2 disruptant strain; $\bigcirc, \operatorname{rec} A$ disruptant strain; $\boldsymbol{\square}, \operatorname{rec} A$ lexA1 double-disruptant strain; $\boldsymbol{\Delta}, \operatorname{rec} A$ lexA2 double-disruptant strain. All strains carried plasmid p12Rhyg, in which the radiation-responsive minimal promoter region ( -57 to -1 upstream of the initiation site) of the pprA gene was located upstream of FL.

DNA-binding ability. Amino acid sequence alignment revealed that the $\mathrm{N}$-terminal region of $D$. radiodurans LexA2 did not possess similarities to the E. coli and D. radiodurans LexA1 proteins (Fig. 2). These results suggested that $D$. radiodurans possesses distinct LexA1- and LexA2binding motifs that differ from the E. coli SOS box (Walker, 1984; Friedberg et al., 1995). D. radiodurans LexA1 and LexA2 may be involved in different gene regulations.

A hypothesis concerning the radiation response mechanism in $D$. radiodurans is presented in Fig. 8 on the basis of the results obtained in the present and previous studies. Unlike E. coli, D. radiodurans LexA1 is not involved in RecA induction following $\gamma$-irradiation (Narumi et al., 2001; Bonacossa de Almeida et al., 2002). Furthermore, neither the lexA1 gene deletion nor the mutation leading to a noncleavable LexA1 affected survival or double-strand breaks repair, suggesting that LexA1 does not play a major role in the radiation response mechanism following $\gamma$-irradiation in D. radiodurans (Jolivet et al., 2005). The results of radioresistance in the lexA1 disruptant strains (Fig. 6) are consistent with these conclusions. A previous study assumed that LexA1 and DRA0074 have similar regulatory functions in the metabolic pathway because a lexA1 dra0074 doubledisruptant strain could not be constructed (Sheng et al., 2004). However, we successfully constructed a lexA1 lexA2 double-disruptant strain in the present study. The induction of RecA was observed in the lexA1 lexA2 double-disruptant 
strain (Fig. 5a), indicating that the two LexA homologues do not exhibit functional overlap in relation to RecA induction. These results support the notion that recA expression following $\gamma$-irradiation is regulated by DR0167 (also called PprI/IrrE) in D. radiodurans (Earl et al., 2002; Hua et al., 2003) (Fig. 8).

In a previous study we suggested that the co-protease activity rather than the recombination activity of RecA contributed to radiation resistance in $D$. radiodurans, given that a $D$. radiodurans recA mutant strain that completely lacked recombination activity but retained co-protease activity of RecA showed only slight sensitivity to $\gamma$-rays compared with the wild-type (Satoh et al., 2002). RecA can accelerate autocleavage of $D$. radiodurans LexA1 (Narumi et al., 2001), and LexA2 autocleavage is also accelerated by RecA (Fig. 4b, lane 6). This suggests that a certain gene down-regulated by LexA1 and/or LexA2 might be important for the radioresistance of $D$. radiodurans.

Sheng et al. (2004) examined the radiosensitivity of dra0074 disruptant strain M74 up to a dose of $8 \mathrm{kGy}$, and found no significant difference in survival curves between strain M74 and the wild-type. However, we found that lexA2 disruptant strains exhibited a much higher resistance to $\gamma$-rays than the wild-type at high doses ( 8 to $15 \mathrm{kGy}$ ) (Fig. 6a). At least part of the increase in radioresistance of the lexA2 disruptant strain may be explained by the enhancement of pprA

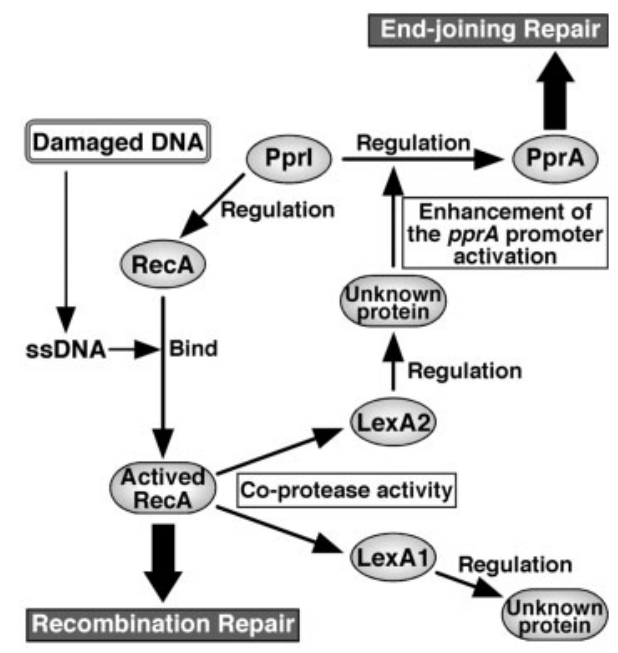

Fig. 8. Hypothesis concerning the radiation response mechanism in D. radiodurans. Following DNA damage, Pprl up-regulates the induction of RecA and PprA (Earl et al., 2002; Hua et al., 2003; Ohba et al., 2005). RecA is activated by binding with ssDNA segments generated from the damaged DNA. Activated RecA serves as a co-protease to stimulate LexA1 (Narumi et al., 2001) and LexA2 cleavages. An unknown protein down-regulated by LexA2 enhances pprA promoter activation. RecA and PprA play crucial roles in recombination and end-joining repairs, respectively (Satoh et al., 2002, Narumi et al., 2004). promoter activation (Fig. 8). D. radiodurans PprA preferentially binds to dsDNA carrying strand breaks (Narumi et al., 2004). DNA strand breaks also result from the process of DNA replication. We suggest that the intracellular PprA level must be regulated. If PprA is induced constitutively, cell growth will be inhibited in D. radiodurans because PprA binds to DNA strand breaks that are generated by DNA replication. Therefore, the regulation by LexA2 contributes to the stable maintenance of cell growth in the undamaged state. While an enhancement of $p p r A$ promoter activation in the recA lexA2 double-disruptant strain was not prominent (Fig. 7), lexA2 disruption did not restore radiosensitivity in the recA background (Fig. 6b). This finding suggested that the radiation response mechanism proceeds in a RecAdependent manner.

\section{ACKNOWLEDGEMENTS}

We thank Y. Kobayashi, M. Kikuchi, T. Funayama and S. Kitayama for valuable discussions. This work was funded in part by a grant to I. Narumi from the Ministry of Education, Culture, Sports, Science and Technology of Japan.

\section{REFERENCES}

Anderson, A. W., Nordan, H. C., Cain, R. F., Parrish, G. \& Duggan, D. (1956). Studies on a radio-resistant Micrococcus. I. Isolation, morphology, cultural characteristics, and resistance to gamma radiation. Food Technol 10, 575-578.

Bateman, A., Coin, L., Durbin, R. \& 10 other authors (2004). The Pfam protein families database. Nucleic Acids Res 32, D138-D141.

Battista, J. R. (1997). Against all odds: the survival strategies of Deinococcus radiodurans. Annu Rev Microbiol 51, 203-224.

Bonacossa de Almeida, C., Costa, G., Sommer, S. \& Bailone, A. (2002). Quantification of RecA protein in Deinococcus radiodurans reveals involvement of RecA, but not LexA, in its regulation. Mol Genet Genomics 268, 28-41.

Cox, M. M. \& Battista, J. R. (2005). Deinococcus radiodurans - the consummate survivor. Nat Rev Microbiol 3, 882-892.

Dean, C. J., Feldschreiber, P. \& Lett, J. T. (1966). Repair of X-ray damage to the deoxyribonucleic acid in Micrococcus radiodurans. Nature 209, 49-52.

Earl, A. M., Mohundro, M. M., Mian, S. \& Battista, J. R. (2002). The IrrE protein of Deinococcus radiodurans $\mathrm{R} 1$ is a novel regulator of recA expression. J Bacteriol 184, 6216-6224.

Fogh, R. H., Ottleben, G., Rüterjans, H., Schnarr, M., Boelens, R. \& Kaptein, R. (1994). Solution structure of the LexA repressor DNA binding domain determined by ${ }^{1} \mathrm{H}$ NMR spectroscopy. EMBO J 13, 3936-3944.

Friedberg, E. C., Walker, G. C. \& Siede, W. (1995). DNA Repair and Mutagenesis. Washington, DC: American Society for Microbiology.

Funayama, T., Narumi, I., Kikuchi, M., Kitayama, S., Watanabe, H. \& Yamamoto, K. (1999). Identification and disruption analysis of the recN gene in the extremely radioresistant bacterium Deinococcus radiodurans. Mutat Res 435, 151-161.

Grimsley, J. K., Masters, C. I., Clark, E. P. \& Minton, K. W. (1991). Analysis by pulsed-field gel electrophoresis of DNA double-strand breakage and repair in Deinococcus radiodurans and a radiosensitive mutant. Int J Radiat Biol 60, 613-626. 
Hansen, M. T. (1980). Four proteins synthesized in response to deoxyribonucleic acid damage in Micrococcus radiodurans. J Bacteriol 141, 81-86.

Horii, T., Ogawa, T. \& Ogawa, H. (1981). Nucleotide sequence of the lexA gene of E. coli. Cell 23, 689-697.

Hua, Y., Narumi, I., Gao, G., Tian, B., Satoh, K., Kitayama, S. \& Shen, B. (2003). PprI: a general switch responsible for extreme radioresistance of Deinococcus radiodurans. Biochem Biophys Res Commun 306, 354-360.

Islam, M. S., Hua, Y., Ohba, H., Satoh, K., Kikuchi, M., Yanagisawa, T. \& Narumi, I. (2003). Characterization and distribution of IS8301 in the radioresistant bacterium Deinococcus radiodurans. Genes Genet Syst 78, 319-327.

Jolivet, E., Lecointe, F., Coste, G., Satoh, K., Narumi, I., Bailone, A. \& Sommer, S. (2005). Limited concentration of RecA delays DNA double strand break repair in Deinococcus radiodurans R1. Mol Microbiol 59, 338-349.

Kikuchi, M., Narumi, I., Kitayama, S., Watanabe, H. \& Yamamoto, K. (1999). Genomic organization of the radioresistant bacterium Deinococcus radiodurans: physical map and evidence for multiple replicons. FEMS Microbiol Lett 174, 151-157.

Kitayama, S. \& Matsuyama, A. (1968). Possibility of the repair of double-strand scissions in Micrococcus radiodurans DNA caused by gamma-rays. Biochem Biophys Res Commun 33, 418-422.

Kitayama, S. \& Matsuyama, A. (1971). Double-strand scissions in DNA of gamma-irradiated Micrococcus radiodurans and their repair during postirradiation incubation. Agric Biol Chem 35, 644-652.

Kitayama, S. \& Matsuyama, A. (1975). Loss of characteristic radiation resistance by mutation of Micrococcus radiodurans. Mutat Res 29, 327-332.

Lipton, M. S., Pasa-Tolic, L., Anderson, G. A. \& 19 other authors (2002). Global analysis of the Deinococcus radiodurans proteome by using accurate mass tags. Proc Natl Acad Sci U S A 99, 11049-11054.

Liu, Y., Zhou, J., Omelchenko, M. V. \& 12 other authors (2003). Transcriptome dynamics of Deinococcus radiodurans recovering from ionizing radiation. Proc Natl Acad Sci U S A 100, 4191-4196.

Luo, Y., Pfuetzner, R. A., Mosimann, S., Paetzel, M., Frey, E. A., Cherney, M., Kim, B., Little, J. W. \& Strynadka, N. C. J. (2001). Crystal structure of LexA: a conformational switch for regulation of self-cleavage. Cell 106, 585-594.

Makarova, K. S., Aravind, L., Wolf, Y. I., Tatusov, R. L., Minton, K. W., Koonin, E. V. \& Daly, M. J. (2001). Genome of the extremely radiation-resistant bacterium Deinococcus radiodurans viewed from the perspective of comparative genomics. Microbiol Mol Biol Rev 65, 44-79.

Mattimore, V., Udupa, K. S., Berne, G. A. \& Battista, J. R. (1995). Genetic characterization of forty ionizing radiation-sensitive strains of Deinococcus radiodurans: linkage information from translation. J Bacteriol 177, 5232-5237.

Mennecier, S., Coste, G., Servant, P., Bailone, A. \& Sommer, S. (2004). Mismatch repair ensures fidelity of replication and recombination in the radioresistant organism Deinococcus radiodurans. Mol Genet Genomics 272, 460-469.

Miller, R. V. \& Kokjohn, T. A. (1990). General microbiology of recA. Environmental and evolutionary significance. Annu Rev Microbiol 44, 365-394.

Moseley, B. E. B. (1967). The isolation and some properties of radiation sensitive mutants of Micrococcus radiodurans. J Gen Microbiol 49, 293-300.
Narumi, I. (2003). Unlocking radiation resistance mechanisms: still a long way to go. Trends Microbiol 11, 422-425.

Narumi, I., Cherdchu, K., Kitayama, S. \& Watanabe, H. (1997). The Deinococcus radiodurans uvrA gene: identification of mutation sites of two mitomycin-sensitive strains and the first discovery of insertion sequence element from deinobacteria. Gene 198, 115-126.

Narumi, I., Satoh, K., Kikuchi, M., Funayama, T., Kitayama, S., Yanagisawa, T., Watanabe, H. \& Yamamoto, K. (1999). Molecular analysis of the Deinococcus radiodurans recA locus and identification of a mutation site in a DNA repair-deficient mutant, rec30. Mutat Res 435, 233-243.

Narumi, I., Satoh, K., Kikuchi, M., Funayama, T., Yanagisawa, T., Kobayashi, Y., Watanabe, H. \& Yamamoto, K. (2001). The LexA protein from Deinococcus radiodurans is not involved in RecA induction following $\gamma$ irradiation. J Bacteriol 183, 6951-6956.

Narumi, I., Satoh, K., Cui, S., Funayama, T., Kitayama, S. \& Watanabe, H. (2004). PprA: a novel protein from Deinococcus radiodurans that stimulates DNA ligation. Mol Microbiol 54, 278-285.

Nishida, H. \& Narumi, I. (2002). Disruption analysis of DR1420 and/ or DR1758 in the extremely radioresistant bacterium Deinococcus radiodurans. Microbiology 148, 2911-2914.

Ohba, H., Satoh, K., Yanagisawa, T. \& Narumi, I. (2005). The radiation responsive promoter of the Deinococcus radiodurans pprA gene. Gene 363, 133-141.

Roca, A. I. \& Cox, M. M. (1990). The RecA protein: structure and function. Crit Rev Biochem Mol Biol 25, 415-456.

Satoh, K., Narumi, I., Kikuchi, M., Kitayama, S., Yanagisawa, T., Yamamoto, K. \& Watanabe, H. (2002). Characterization of RecA424 and RecA670 proteins from Deinococcus radiodurans. J Biochem 131, 121-129.

Sheng, D., Zheng, Z., Tian, B., Shen, B. \& Hua, Y. (2004). LexA analog (dra0074) is a regulatory protein that is irrelevant to $r e c A$ induction. J Biochem 136, 787-793.

Tanaka, A., Hirano, H., Kikuchi, M., Kitayama, S. \& Watanabe, H. (1996). Changes in cellular proteins of Deinococcus radiodurans following $\gamma$-irradiation. Radiat Environ Biophys 35, 95-99.

Tanaka, M., Earl, A. M., Howell, H. A., Park, M. J., Eisen, J. A., Peterson, S. N. \& Battista, J. R. (2004). Analysis of Deinococcus radiodurans's transcriptional response to ionizing radiation and desiccation reveals novel proteins that contribute to extreme radioresistance. Genetics 168, 21-33.

Thompson, J. D., Higgins, D. G. \& Gibson, T. J. (1994). CLUSTAL W: improving the sensitivity of progressive multiple sequence alignment through sequence weighting, position-specific gap penalties and weight matrix choice. Nucleic Acids Res 22, 4673-4680.

Walker, G. C. (1984). Mutagenesis and inducible responses to deoxyribonucleic acid damage in Escherichia coli. Microbiol Rev 48, 60-93.

White, O., Eisen, J. A., Heidelberg, J. F. \& 29 other authors (1999). Genome sequence of the radioresistant bacterium Deinococcus radiodurans $\mathrm{R} 1$. Science 286, 1571-1577.

Wojciechowski, M. F., Peterson, K. R. \& Love, P. E. (1991). Regulation of the SOS response in Bacillus subtilis: evidence for a LexA repressor homolog. J Bacteriol 173, 6489-6498.

Zhang, C., Wei, J., Zheng, Z., Ying, N., Sheng, D. \& Hua, Y. (2005). Proteomic analysis of Deinococcus radiodurans recovering from $\gamma$ irradiation. Proteomics 5, 138-143. 